\title{
Global Attractor for a Chemotaxis Model with Reaction Term
}

\author{
Xueyong Chen and Jianwei Shen \\ School of Mathematics and Statistics, Xuchang University, Xuchang 461000, China \\ Correspondence should be addressed to Jianwei Shen; xcjwshen@gmail.com
}

Received 15 December 2012; Revised 19 June 2013; Accepted 19 June 2013

Academic Editor: Marco H. Terra

Copyright (C) 2013 X. Chen and J. Shen. This is an open access article distributed under the Creative Commons Attribution License, which permits unrestricted use, distribution, and reproduction in any medium, provided the original work is properly cited.

A chemotaxis model with reproduction term in a bounded domain $\Omega \subset R^{n}$ is discussed in this paper, where $n=2,3$. First, the existence of a global-in-time solution is given, and then a global attractor for this model is obtained.

\section{Introduction}

This paper deals with global attractor of a quasilinear parabolic system introduced in [1] by Horstmann and Winkler to model chemotaxis. Chemotaxis phenomenon is quite common in biosystem. The survival of many organisms, from microscopic bacteria to the largest mammals, depends on their ability to navigate in a complex environment through the detection, integration, and processing of a variety of internal and external signals. This movement is crucial for many aspects of behavior, including the location of food sources, avoidance of predators, and attracting mates. The ability to migrate in response to external signals is shared by many cell populations. The directed movement of cells and organisms in response to chemical gradients is called chemotaxis.

The classical chemotaxis model has been extensively studied in the last few years (see [1-5]); Payne and Straughan [4] tackle precise nonlinear decay for classical model. Decay for a nonlinear chemotaxis system modeling glia cell movement in the brain is treated by Quinlan and Straughan [5].

In this paper, we are concerned with the following chemotaxis model:

$$
\begin{aligned}
& \frac{\partial u}{\partial t} \\
& =\Delta u-\nabla \cdot\left((u+1)^{\alpha} \nabla g(w)\right)+f(u, w), \quad x \in \Omega, t>0, \\
& \frac{\partial w}{\partial t}=\Delta w+u-w, \quad x \in \Omega, t>0,
\end{aligned}
$$

$$
\begin{gathered}
\frac{\partial u}{\partial n}=\frac{\partial w}{\partial n}=0, \quad x \in \partial \Omega, \\
u(x, 0)=u_{0}(x), \quad w(x, 0)=w_{0}(x), \quad x \in \bar{\Omega},
\end{gathered}
$$

where $u(x, t)$ represents the density of a biological species, which could be a cell, a germ, or an insect, while $w(x, t)$ denotes the concentration of a chemical substance on position $x \in \Omega$ and at time $t \in[0, \infty) . \alpha$ is a positive constant. $\Omega$ is an open bounded subset of $R^{n}$ with the boundary of class $C^{3} \cdot g(s), f(s)$ are two real smooth functions for $s \in[0,+\infty)$ satisfying the following conditions:

(i) $f(0, w)=0$;

(ii) there exists $M>0$ such that $f(u, w) \leq 0$ as $u>M$;

(iii) there exists $\lambda>0$ such that $\left|g^{\prime}(s)\right|<\lambda$ for all $s \in$ $[0,+\infty)$.

$g^{\prime}(w)(u+1)^{\alpha}$ denotes a chemotactic sensitivity function. $(u+1)^{\alpha}$ can be replaced by $(u+\varepsilon)^{\alpha}$, where $\varepsilon$ is an arbitrary positive constant. For convenience, we choose $\varepsilon=1 . f(u, w)$ describes the intrinsic rate of growth of cells population. For $\alpha=1, \varepsilon=0$, and $g(w)=w$, system (1) equals the most common formulation of the Keller-Segel model.

To our knowledge it has never been analyzed whether the global attractor of system (1) exists if the positive power $\alpha \neq 1$; this system is more motivated from the mathematical point of view than from the biological one, but it will help to get more 
insights in the understanding of the behavior of the problem. We will look at this aspect of the critical exponent $\alpha$ which decides whether global attractor can exist or not.

The existence of the global attractor for semilinear reaction diffusion equations in bounded and unbounded domains has been studied extensively $[6,7]$. However, chemotaxis model is always a strongly coupled quasilinear parabolic system. There are a few articles to discuss the global attractor of such a system. We construct a local solution to (1) by the semigroup method and then discuss its regularity by a priori estimate method we set up for a strongly coupled quasilinear parabolic system.

For readers' convenience, the following standard result on attractor is first presented here (see [3, 6-9]).

Proposition 1. Suppose that $X$ is a metric space and $S(t)_{t \geq 0}$ is a semigroup of continuous operators in $X$. If $S(t)_{t \geq 0}$ has a bounded absorbing set and is asymptotically compact, then $S(t)_{t \geq 0}$ possesses a global attractor which is a compact invariant set and attracts every bounded set in $X$.

Definition 2 . The semigroup $S(t)$ is asymptotically compact; that is, if $u_{n}$ is bounded in $X$ and $t_{n} \rightarrow \infty$, then $S\left(t_{n}\right) u_{n}$ is precompact in $X$.

We first show that there is a unique global solution to (1) for any nonnegative initial functions $u_{0}(x) \in L^{2}(\Omega), w_{0}(x) \in$ $W^{1,2}(\Omega)$. And then we define a continuous semigroup $S(t)_{t \geq 0}$ in $L^{2}(\Omega) \times W^{1,2}(\Omega)$. Next, we find a bounded absorbing set and prove that the semigroup $S(t)$ is asymptotically compact. Thus, from the standard result, it is shown that there is a global attractor to $(1)$ in $L^{2}(\Omega) \times W^{1,2}(\Omega)$.

\section{Preliminary}

Some well-known inequalities and embedding results that will be used in the sequel are presented.

Lemma 3 (see [10]). If $p, q \geq 1$ and $p(n-q)<n q$, then, for $r \in(0, p)$,

$$
\|u\|_{L^{p}(\Omega)} \leq c\|u\|_{W^{1, q}}^{a} \cdot\|u\|_{L^{r}(\Omega)}^{(1-a)} \quad \forall u \in W^{1, q}(\Omega),
$$

where $a=(n / r-n / p) /(1-n / q+n / r) \in(0,1)$.

Lemma 4 (see $[10]$ ). Let $1 \leq q \leq p \leq \infty$ and $f \in L^{q}(\Omega)$. Then

$$
\begin{gathered}
\left\|e^{t \Delta} f\right\|_{p} \leq(4 \pi t)^{(-n / 2)(1 / q-1 / p)}\|f\|_{q} \\
\left\|\nabla e^{t \Delta} f\right\|_{p} \leq c t^{(-n / 2)(1 / q-1 / p)-1 / 2}\|f\|_{q} \\
\left\|(-\Delta+1)^{\beta} e^{t \Delta} f\right\|_{p} \leq c t^{-\beta(-n / 2)(1 / q-1 / p)} e^{(1-\mu) t}\|f\|_{q} \quad p \neq q,
\end{gathered}
$$

where $\beta>0, \mu>0$, and $c$ is a positive constant depending only on $p, q, \Omega$.

$\left(e^{t \Delta} f\right)(x)=\int_{\Omega} G(x-y, t) f(y) d y$ and $G(x, t)$ is the Green function of the heat equation $\partial u / \partial t-\Delta u=0, x \in \Omega, t>0$ with the homogeneous Neumann boundary condition $\partial u / \partial n=0$.
Lemma 5 (see [11]). Let $A_{p}=-\triangle$ and $D\left(A_{p}\right)=\{\varphi \in$ $\left.W_{(\Omega)}^{2, p}|\partial \varphi / \partial N|_{\partial \Omega}=0\right\}$. Then

$$
\begin{gathered}
D\left(\left(A_{p}+1\right)^{\beta}\right) \hookrightarrow W^{1, p}(\Omega), \quad \text { if } \beta>\frac{1}{2}, \\
D\left(\left(A_{p}+1\right)^{\beta}\right) \hookrightarrow C^{\delta}(\Omega), \quad \text { if } 2 \beta-\frac{n}{p}>\delta \geq 0, \\
\left\|(A+1)^{\beta} e^{-t(A+1)} u\right\|_{L^{p}(\Omega)} \leq c t^{-\beta}\|u\|_{L^{p}(\Omega)} .
\end{gathered}
$$

Lemma 6. Let $r$ and $s$ be nonnegative real numbers satisfying $r+s<2$. Then for any $\varepsilon>0$ there exists a constant $c_{\varepsilon}>0$ such that

$$
a^{r} b^{s} \leq \varepsilon\left(a^{2}+b^{2}\right)+c_{\varepsilon} \quad \forall a, b>0 .
$$

Lemma 7 (see [2]). Suppose that $H^{s}$ is an interpolation space of $H^{s_{0}}$ and $H^{s_{1}}$, where $0 \leq s_{0}<s_{1}<\infty, 0<\theta<1, s=$ $(1-\theta) s_{0}+\theta s_{1}$, and then

$$
\|\cdot\|_{H^{s}} \leq c\|\cdot\|_{H^{s_{0}}}^{1-\theta}\|\cdot\|_{H^{s_{1}}}^{\theta} .
$$

Here $H^{s}(\Omega), s>0$, denotes the fractional Sobolev space in $\Omega$.

Lemma 8 (see [1]). Let $\beta>0, p \in(1,+\infty)$, for all $\varepsilon>0$; there exists $c(\varepsilon)>0$ for any $w \in L^{p}(\Omega)$, such that

$$
\left\|(A+1)^{\beta} e^{-t A} \nabla \cdot w\right\|_{L_{(\Omega)}^{p}} \leq c(\varepsilon) t^{-\beta-(1 / 2)-\varepsilon}\|w\|_{L_{(\Omega)}^{p}} .
$$

\section{Local Existence and Uniqueness}

The local existence of a solution to system (1) is discussed in this section. First, an estimate to $w$ in (1) is shown.

Lemma 9. Assume that there exist $1 \leq q \leq p \leq \infty,(1 / q)-$ $(1 / p)<1 / n$, and $u \in L^{\infty}\left([0, \infty) ; L^{q}(\Omega)\right)$. Then for any $\tau>0$

$$
\|w(t)\|_{W^{1, p}} \leq \tau^{-\alpha}\left\|w_{0}\right\|_{L^{1}}+c \Gamma(\gamma) \sup _{\tau<s<t}\|u(s)\|_{q} \quad \forall t \geq \tau,
$$

where $\alpha>0, \gamma=1-\beta-(n / 2)((1 / q)-(1 / p)), \Gamma(\cdot)$ is the Gamma function, and

$$
w(x, t)=e^{t(\Delta-1)} w_{0}+\int_{0}^{t} e^{(t-s)(\Delta-1)} u(s) d s
$$

The proof of the lemma can be found in another paper [3].

Theorem 10. Suppose $0 \leq u_{0}(x) \in L^{2}(\Omega), 0 \leq w_{0}(x) \epsilon$ $W^{1,2}(\Omega)$, and $\alpha<1 / n$. Then there is a $T \leq \infty$ (depending on $\left.\left\|u_{0}\right\|_{L^{2}(\Omega)},\left\|w_{0}\right\|_{W^{1,2}(\Omega)}\right)$ such that there exists a unique nonnegative solution $(u(x, t), w(x, t))$ to $(1)$ in $[0, T]$ and

$$
\begin{aligned}
u \in & C^{0}\left([0, T] ; L^{2}(\Omega)\right) \bigcap C^{2,1}(\Omega ;(0, T]), \\
w \in & C^{0}\left([0, T] ; W^{1,2}(\Omega)\right) \bigcap C^{2,1}(\Omega ;(0, T]) \\
& \bigcap C^{0}\left((0, T] ; C^{3}(\Omega)\right) .
\end{aligned}
$$


Proof. Choose $T \in(0,1)$ and $R>0$ to be fixed. In Banach space $X=C^{0}\left([0, T] ; L^{2}(\Omega)\right) \times C^{0}\left([0, T] ; W^{1,2}(\Omega)\right)$, we define a bounded closed set

$$
S:=\left\{(u, w) \in X \mid\|u, w\|_{X} \leq R\right\} .
$$

Let

$$
\begin{aligned}
\psi(u, w)(t) & \\
& =\left(\begin{array}{l}
\psi_{1}(u, w)(t) \\
\psi_{2}(u, w)(t)
\end{array}\right) \\
& =\left(\begin{array}{c}
e^{t \Delta} u_{0}-\int_{0}^{t} e^{(t-s) \Delta}\left[\nabla\left(u^{\alpha} \nabla g(w)\right)+f(u)\right] d s \\
e^{t(\Delta-1)} w_{0}+\int_{0}^{t} e^{(t-s)(\Delta-1)} u(s) d s
\end{array}\right),
\end{aligned}
$$

where $f(u)=u(a-b u)$.

Next, we prove that $\psi$ is a contractive mapping from $S$ into itself for $T$ small enough and $R$ sufficiently large. By Lemma 4 , then

$$
\begin{aligned}
& \left\|\psi_{1}(u, w)\right\|_{L^{2}} \\
& \leq\left\|e^{-t A} u_{0}(x)\right\|_{L^{2}}+c \int_{0}^{t}\left\|e^{-(t-s) A} \nabla\left(u^{\alpha} \nabla g(w)\right)\right\|_{L^{2}} d s \\
& \quad+\int_{0}^{t}\left\|e^{-(t-s) A} f(u(s))\right\|_{L^{2}} s s \\
& \leq\left\|u_{0}(x)\right\|_{L^{2}} \\
& \quad+c \int_{0}^{t}(t-s)^{(-n / 2)((1 / q)-(1 / 2))-(1 / 2)}\left\|u^{\alpha} \nabla g(w)\right\|_{L^{q}} d s \\
& \quad+\int_{0}^{t}(t-s)^{(-n / 2)(1-(1 / 2))}\|f(u(s))\|_{L^{1}} d s,
\end{aligned}
$$

where $A=-\triangle$. Since $\alpha<1 / n$, there is a $q \in[2 n /(2+n), 2)$, such that $2 q \alpha /(2-q) \leq 2$ and $1 / 2-(n / 2)((1 / q)-(1 / 2))>0$. By Hölder's inequality

$$
\begin{aligned}
\left\|u^{\alpha} \nabla g(w)\right\|_{L^{q}} & \leq \lambda\left\|u^{\alpha} \nabla w\right\|_{L^{q}} \leq c(\lambda)\|u\|_{L^{2 q \alpha /(2-q)}}^{\alpha}\|\nabla w\|_{L^{2}} \\
& \leq c(\lambda)\|u\|_{L^{2}}^{\alpha}\|\nabla w\|_{L^{2}} \\
& \leq c(\lambda) R^{2},
\end{aligned}
$$

then

$$
\begin{aligned}
& c \int_{0}^{t}(t-s)^{(-n / 2)((1 / q)-(1 / 2))-(1 / 2)}\left\|u^{\alpha} \nabla g(w)\right\|_{L^{q}} d s \\
& \quad \leq c(\lambda) R^{2} T^{(1 / 2)-(n / 2)((1 / q)-(1 / 2))} .
\end{aligned}
$$

Similarly, for $n \leq 3$, there is $\int_{0}^{t}(t-$ $s)^{(-n / 2)(1-(1 / 2))}\|f(u(s))\|_{L^{1}} d s \leq R T^{1-(n / 4)}$. Hence

$$
\left\|\psi_{1}(u, w)\right\|_{L^{2}} \leq\left\|u_{0}(x)\right\|_{L^{2}}+c R^{2} T^{1 / 2-(n / 2)((1 / q)-(1 / 2))}
$$$$
+R T^{1-(n / 4)} .
$$

By Lemma 5 , for any $t \in[0, T)$, there is a $\gamma \in(1 / 2,1)$ such that

$$
\begin{aligned}
& \left\|\psi_{2}(u, w)\right\|_{W^{1,2}} \\
& \leq\left\|e^{-t(A+1)} w_{0}(x)\right\|_{W^{1,2}}+\int_{0}^{t}\left\|e^{-(t-s)(A+1)} u(s)\right\|_{W^{1,2}} d s \\
& \leq\left\|w_{0}(x)\right\|_{W^{1,2}}+c \int_{0}^{t}\left\|(A+1)^{\gamma} e^{-(t-s)(A+1)} u(s)\right\|_{L^{2}} d s \\
& \leq\left\|w_{0}(x)\right\|_{W^{1,2}}+c \int_{0}^{t}(t-s)^{-\gamma}\|u(s)\|_{L^{2}} d s \\
& \leq\left\|w_{0}(x)\right\|_{W^{1,2}}+c R T^{1-\gamma} .
\end{aligned}
$$

Equations (16) and (17) imply that $\psi S \subset S$ for any fixed positive $R$ large enough and $T$ small enough. Now we show that $\psi$ is a contractive operator from $S$ to $S$. For for all $(u, w)$, $(\bar{u}, \bar{w}) \in S$,

$$
\begin{aligned}
& \left\|\psi_{1}(u, w)-\psi_{1}(\bar{u}, \bar{w})\right\|_{L^{2}} \\
& \leq c \int_{0}^{t}\left\|e^{-(t-s) A}\left[\nabla\left(u^{\alpha} \nabla g(w)\right)-\nabla\left(\bar{u}^{\alpha} \nabla g(\bar{w})\right)\right]\right\|_{L^{2}} d s \\
& \quad+\int_{0}^{t}\left\|e^{-(t-s) A}(f(u)-f(\bar{u}))\right\|_{L^{2}} d s \\
& \leq c \int_{0}^{t}(t-s)^{(-n / 2)((1 / q)-(1 / 2))-(1 / 2)}\left\|u^{\alpha} \nabla w-\bar{u}^{\alpha} \nabla \bar{w}\right\|_{L^{q}} d s \\
& \quad+\int_{0}^{t}(t-s)^{-n / 4}\|f(u)-f(\bar{u})\|_{L^{1}} d s \\
& \leq c R^{2}\left(T^{1 / 2-(n / 2)((1 / q)-(1 / 2))}+T^{1-n / 4}\right)\|(u, w)-(\bar{u}, \bar{w})\|_{X} \\
& \left\|\psi_{2}(u, w)-\psi_{2}(\bar{u}, \bar{w})\right\|_{W^{1,2}} \\
& \leq c \int_{0}^{t}\left\|(A+1)^{\gamma} e^{-(t-s)(A+1)}(u(s)-\bar{u}(s))\right\|_{L^{2}} d s \\
& \leq c \int_{0}^{t}(t-s)^{-\gamma}\|u(s)-\bar{u}(s)\|_{L^{2}} d s \\
& \leq c T^{1-\gamma}\|(u, w)-(\bar{u}, \bar{w})\|_{X} \quad \forall t \in[0, T) . \\
& \quad \forall t \in[0, T)
\end{aligned}
$$

Equation (18) implies that $\psi$ is a contractive mapping if $T$ is sufficiently small. By Banach's fixed point theorem, there exists a unique fixed point $(u, w) \in X$ which is just a local solution to (1) in $X$. 
Since $u_{0}(x) \geq 0, w_{0}(x) \geq 0$, then, for any given smooth function $w, u=0$ is the subsolution of the following problem:

$$
\begin{array}{r}
\frac{\partial u}{\partial t}-\Delta u+\nabla \cdot\left((u+1)^{\alpha} \nabla g(w)\right)-u(a-b u)=0, \\
x \in \Omega, t>0, \\
\frac{\partial u}{\partial n}=0, \quad x \in \partial \Omega, \\
u(x, 0)=u_{0}(x), \quad x \in \bar{\Omega}
\end{array}
$$

and, for any given smooth function $u, w=0$ is the subsolution of the following problem:

$$
\begin{gathered}
\frac{\partial w}{\partial t}-\Delta w+w=u, \quad x \in \Omega, t>0, \\
\frac{\partial w}{\partial n}=0, \quad x \in \partial \Omega \\
w(x, 0)=w_{0}(x), \quad x \in \bar{\Omega} .
\end{gathered}
$$

By the comparison principle, for any $t \in\left[0, T_{\max }\right)$ and $x \in \bar{\Omega}$, $u(x, t) \geq 0 ; w(x, t) \geq 0$.

Now, we discuss the regularity of the solution to (1). From the previous analysis, $u(t, x)$ is bounded in $L^{2}(\Omega)$ for any $t \epsilon$ $[0, T]$. Then by Lemma 9 , for any $\tau \in(0, T]$, we deduce that $w(t) \in W^{1, p}(\Omega)$, for all $t \in[\tau / 2, T]$, where $n<p<2 n /(n-$ 2 ) and $n=2$, 3. From the semigroup representation of the solution to (1) and Lemmas 3 and 4 , for any $q \in(2, n /(n-2))$, $t \in[\tau, T]$,

$$
\begin{aligned}
& \|u(x, t)\|_{L^{q}} \\
& \leq\left\|e^{-(t-\tau / 2) A} u_{\tau / 2}(x)\right\|_{L^{q}} \\
& \quad+c \int_{\tau / 2}^{t}\left\|e^{-(t-s) A} \nabla\left((u+1)^{\alpha} \nabla g(w)\right)\right\|_{L^{q}} d s \\
& \quad+\int_{\tau / 2}^{t}\left\|e^{-(t-s) A} f(u(s))\right\|_{L^{q}} d s \\
& \leq\left(t-\frac{\tau}{2}\right)^{(-n / 2)((1 / 2)-(1 / q))}\left\|u_{\tau / 2}(x)\right\|_{L^{2}} \\
& \quad+c \lambda \int_{\tau / 2}^{t}(t-s)^{(-n / 2)((1 / 2)-(1 / q))}\left\|(u+1)^{\alpha} \nabla w\right\|_{L^{2}} d s \\
& \quad+\int_{\tau / 2}^{t}(t-s)^{(-n / 2)(1-(1 / q))}\|f(u(s))\|_{L^{1}} d s .
\end{aligned}
$$

Since $\alpha<1 / n$, then there exists $p_{1} \in(n / 2,1 / \alpha]$ such that $2 \alpha p_{1} \leq 2,2 p_{1}^{\prime}<2 n /(n-2)$ and

$$
\begin{aligned}
& \left\|(u+1)^{\alpha} \nabla w\right\|_{L^{2}} \\
& \quad \leq c\|u\|_{L^{2 \alpha p_{1}}}^{\alpha}\|\nabla w\|_{L^{2 p_{1}^{\prime}}} \leq c\|u\|_{L^{2}}^{\alpha}\|\nabla w\|_{L^{2 p_{1}^{\prime}}} .
\end{aligned}
$$

Here $1 / p_{1}+1 / p_{1}^{\prime}=1$. Then

$$
\begin{aligned}
& \|u(x, t)\|_{L^{q}} \\
& \quad \leq c \tau^{(-n / 2)((1 / 2)-(1 / q))}\left\|u_{0}(x)\right\|_{L^{2}} \\
& \quad+c \sup _{\tau / 2<s<t}\|u\|_{L^{2}}\|\nabla w\|_{L^{2 p_{1}^{\prime}}} \int_{\tau / 2}^{t}(t-s)^{(-n / 2)((1 / 2)-(1 / q))-(1 / 2)} d s \\
& \quad+c \int_{\tau / 2}^{t}(t-s)^{(-n / 2)(1-1 / q)} d s \quad t \in[\tau, T]
\end{aligned}
$$

which implies that, for any $q \in(2, n /(n-2)),\|u(x, t)\|_{L^{q}}$ is bounded for all $t \in[\tau, T]$. By using Lemma 9 again and repeating the above process, it can be proved that

$$
u \in C^{0}\left((\tau, T] ; L^{\infty}(\Omega)\right), \quad w \in C^{0}\left((\tau, T] ; C^{0}(\Omega)\right) .
$$

There exist $p, \beta$, and $\varepsilon$ satisfying $p>n, 1 / 2>\beta>n / 2 p$, and $\varepsilon<1 / 2-\beta$ such that, for any small enough constant $\eta>0$,

$$
\begin{aligned}
\|u(x, t)\|_{C^{0}} & \\
\leq & \left\|e^{-(t-\tau) A} u_{\tau}(x)\right\|_{C^{0}} \\
& +c \int_{\tau}^{t}\left\|e^{-(t-s) A} \nabla\left((u+1)^{\alpha} \nabla g(w)\right)\right\|_{C^{0}} d s \\
& +\int_{\tau}^{t}\left\|e^{-(t-s) A} f(u(s))\right\|_{C^{0}} d s \\
\leq & \left\|(A+1)^{\beta} e^{-(t-\tau) A} u_{\tau}(x)\right\|_{L^{p}} \\
& +c \lambda \int_{\tau}^{t}\left\|(A+1)^{\beta} e^{-(t-s) A} \nabla\left((u+1)^{\alpha} \nabla w\right)\right\|_{L^{p}} d s \\
& +\int_{\tau}^{t}\left\|(A+1)^{\beta} e^{-(t-s) A} f(u(s))\right\|_{L^{p}} d s \\
\leq & \eta^{(-n / 2)((1 / 2)-(1 / p))} e^{(1-\mu) t}\left\|u_{\tau}(x)\right\|_{L^{2}} \\
& +c \sup _{\tau<s<t}\left\|(u+1)^{\alpha} \nabla w\right\|_{L^{p}} \int_{\tau}^{t}(t-s)^{-\beta-1 / 2-\varepsilon} d s \\
& +c \int_{\tau}^{t}(t-s)^{-\beta-\varepsilon_{1}} d s \quad t \in[\tau+\eta, T),
\end{aligned}
$$

where $\varepsilon_{1}=(n / 2)((1 / q)-(1 / p)) \leq 1-\beta$, which implies that $u \in C^{0}\left((0, T] ; C^{0}(\Omega)\right)$. In conclusion, we see that

$$
\begin{gathered}
u(x, t) \in C\left([0, T] ; L^{2}(\Omega)\right) \bigcap C^{2,1}(\Omega ;(0, T]), \\
w(x, t) \in C\left([0, T] ; W^{1,2}(\Omega)\right) \bigcap C^{2,1}(\Omega ;(0, T]) \\
\bigcap C\left((0, T] ; C^{3}(\Omega)\right) .
\end{gathered}
$$


By semigroup techniques and Schauder estimates (Theorem IV. 5.1-5.3 in [7] and Lemmas 3.2-3.3 in [10]), we have that

$$
\begin{gathered}
u \in C^{0}\left([0, T] ; L^{2}(\Omega)\right) \bigcap C^{0}\left((0, T] ; H_{N}^{2}(\Omega)\right), \\
w \in C^{0}\left([0, T] ; W^{1,2}(\Omega)\right) \bigcap C^{0}\left((0, T] ; H_{N}^{3}(\Omega)\right) .
\end{gathered}
$$

Here we denote that $H_{N}^{s}=\left\{u \in H^{s}(\Omega) ; \partial u / \partial n=0\right.$, on $\left.\partial \Omega\right\}$. The proof of Theorem 10 is completed.

\section{Global Solution and Some A Priori Estimates}

In this section, the global-in-time existence of a solution to system (1) is proved. The following a priori estimates will play a crucial role in the proof of our result.

Lemma 11. Suppose that $0 \leq u_{0} \in L^{2}(\Omega),(u, v)$ is a solution to (1), and then there exists a positive constant $c_{0}$ such that

$$
\|u(t)\|_{1} \leq c_{0}, \quad \forall t>0
$$

Proof. Integrating the first equation of (1) on $\Omega$, we obtain

$$
\begin{aligned}
\frac{d\|u(t)\|_{1}}{d t} & =\int_{\Omega}\left(a u-b u^{2}\right) d x=a\|u\|_{1}-b \int_{\Omega} u^{2} d x \\
& \leq a\|u\|_{1}-\frac{b}{|\Omega|}\|u\|_{1}^{2} .
\end{aligned}
$$

From the above equation, it is easy to know that $d\|u(t)\|_{1} / d t<$ 0 if $\|u(t)\|_{1} \geq a|\Omega| / b$. Then there exists a constant $c_{0}$ (depending on $a, b$, and $\Omega$ ) such that $\|u(t)\|_{1} \leq c_{0}$.

Lemma 12. Suppose that $0 \leq u_{0} \in L^{2}(\Omega), 0 \leq w_{0} \in W^{1,2}(\Omega)$, and $\alpha<1 / n ;(u, w)$ is a local solution to $(1)$ in $[0, T]$ satisfying

$$
\begin{aligned}
u \in & C^{0}\left([0, T] ; L^{2}(\Omega)\right) \bigcap C^{0}\left((0, T] ; H^{1}(\Omega)\right) \\
& \bigcap C^{0}\left((0, T] ; H_{N}^{2}(\Omega)\right), \\
w \in & C^{0}\left([0, T] ; W^{1,2}(\Omega)\right) \bigcap C^{0}\left((0, T] ; H_{N}^{2}(\Omega)\right) \\
& \bigcap C^{0}\left((0, T] ; H_{N}^{3}(\Omega)\right) .
\end{aligned}
$$

Then for any $\tau>0$,

$$
\begin{gathered}
\|u(t)\|_{L^{2}}^{2}+\|w(t)\|_{W^{1,2}}^{2} \leq c\left(1+e^{-v t}\left(\left\|u_{0}\right\|_{L^{2}}^{2}+\left\|w_{0}\right\|_{W^{1,2}}^{2}\right)\right), \\
0 \leq t \leq T, \\
\|u(t)\|_{H^{1}}+\|w(t)\|_{H^{2}} \leq C, \quad \tau<t \leq T,
\end{gathered}
$$

where $C$ depends only on $\Omega, u_{\tau}, w_{\tau}$, and $v>0$.
Proof. In the process of the proof, we denote any positive constant by $c$ which may change from line to line and let $\tau$ be a small enough constant.

Step 1. Taking the inner product of the first equation of (1) with $u$ in $L^{2}(\Omega)$,

$$
\begin{aligned}
& \frac{1}{2} \frac{d\|u(t)\|_{L^{2}}^{2}}{d t}+\int_{\Omega}|\nabla u|^{2} d x \\
& \quad=\int_{\Omega} u^{\alpha} \nabla u \cdot \nabla g(w) d x+\int_{\Omega} u\left(a u-b u^{2}\right) d x \\
& \quad \leq \frac{1}{2} \int_{\Omega}|\nabla u|^{2} d x+\frac{1}{2} \int_{\Omega} u^{2 \alpha}|\nabla w|^{2} d x+\frac{a^{2}}{4 b} \int_{\Omega} u d x
\end{aligned}
$$

By Hölder's inequality,

$$
\begin{aligned}
\int_{\Omega} u^{2 \alpha}|\nabla w|^{2} d x & \leq\left(\int_{\Omega} u^{2 p \alpha} d x\right)^{1 / p}\left(\int_{\Omega}|\nabla w|^{2 p^{\prime}} d x\right)^{1 / p^{\prime}} \\
& =\|u\|_{L^{2 p \alpha}}^{2 \alpha}\|\nabla w\|_{L^{2 p^{\prime}}}^{2} \quad\left(\frac{1}{p}+\frac{1}{p^{\prime}}=1\right) .
\end{aligned}
$$

If $2 p \alpha(n-2) \leq 2 n$ and $2 p \alpha>1$, then, from Lemma 3 , there exists $a=(n-(n / 2 p \alpha)) /(1+(n / 2)) \in(0,1)$ such that

$$
\|u\|_{L^{2 p \alpha}}^{2 \alpha} \leq\|u\|_{W^{1,2}}^{2 a \alpha}\|u\|_{L^{1}}^{2 \alpha(1-a)} .
$$

Lemmas 9 and 11 imply that $\|\nabla w(t)\|_{q}$ is bounded for any $q \epsilon$ $(1, n /(n-1))$.

If $q<n /(n-1)<2 p^{\prime}<2 n /(n-2)$, then there exists $b=\left((n / q)-\left(n / 2 p^{\prime}\right)\right) /(1-(n / 2)+(n / q)) \in(0,1)$ such that

$$
\|\nabla w\|_{L^{2 p^{\prime}}}^{2} \leq c\|\nabla w\|_{W^{1,2}}^{2 b}\|\nabla w\|_{L^{q}}^{2(1-b)} \leq c\|\Delta w\|_{L^{2}}^{2 b} .
$$

Let $t=1 / p^{\prime}>(n-2) / n, g(\alpha, p, q)=2 a \alpha+2 b$ since $\alpha<1 / n$,

$$
\begin{aligned}
& g\left(\alpha, p, \frac{n}{-1}\right) \\
& \quad=\frac{4 n \alpha-2 n(1-t)}{n+2}+\frac{2}{n}(2 n-2-n t) \\
& \quad=\frac{(4 \alpha+2) n^{2}+4 n-8}{n(n+2)}+\frac{-4 t}{n+2} \\
& \quad<\frac{(4 \alpha+2) n^{2}}{n(n+2)}<2 .
\end{aligned}
$$

Then there exist $p, q$ which satisfy the above condition such that $2 a \alpha+2 b<2$. By Lemma 6 , for any $\varepsilon>0$, there is a constant $c_{\varepsilon}$ such that

$$
\begin{aligned}
& \int_{\Omega} u^{2 \alpha}|\nabla w|^{2} d x \\
& \quad \leq\|\nabla u(t)\|_{L^{2}}^{2 a \alpha}\|\Delta w(t)\|_{L^{2}}^{2 b} \\
& \quad \leq \varepsilon\left(\|\nabla u(t)\|_{L^{2}}^{2}+\|\Delta w(t)\|_{L^{2}}^{2}\right)+c_{\varepsilon} .
\end{aligned}
$$


Taking the inner product of the second equation of (1) with $\triangle w$ in $L^{2}(\Omega)$, for any $\varepsilon_{1}>0$,

$$
\begin{aligned}
& \frac{1}{2} \frac{d\|\nabla w(t)\|_{L^{2}}^{2}}{d t} \\
& \quad+\int_{\Omega}|\Delta w|^{2} d x+\int_{\Omega}|\nabla w|^{2} d x=-\int_{\Omega} u \Delta w d x \\
& \quad \leq \frac{\varepsilon_{1}^{2}}{2} \int_{\Omega}|\nabla u|^{2} d x+\frac{1}{2 \varepsilon_{1}^{2}} \int_{\Omega}|\nabla w|^{2} d x .
\end{aligned}
$$

From (32) and (38), there are $\varepsilon, \varepsilon_{1}$ such that $v=\min \{1-\varepsilon-$ $\left.\varepsilon_{1}^{2}, 2-1 / \varepsilon_{1}^{2}\right\}>0$ and

$$
\begin{aligned}
& \frac{d\left(\|\nabla w(t)\|_{L^{2}}^{2}+\|u(t)\|_{L^{2}}^{2}\right)}{d t} \\
& \quad+v\left(\int_{\Omega}|\Delta w|^{2} d x+\int_{\Omega}|\nabla w|^{2} d x+\int_{\Omega}|\nabla u|^{2} d x\right) \leq c .
\end{aligned}
$$

By the Poincaré inequality,

$$
\int_{\Omega}|\nabla u|^{2} d x \geq c \int_{\Omega} u^{2} d x, \quad \int_{\Omega}|\Delta w|^{2} d x \geq c \int_{\Omega}|\nabla w|^{2} d x
$$

Gronwall's lemma implies that

$$
\begin{array}{r}
\|u(t)\|_{L^{2}}^{2}+\|w(t)\|_{W^{1,2}}^{2} \leq c\left(1+e^{-v t}\left(\left\|u_{0}\right\|_{L^{2}}^{2}+\left\|w_{0}\right\|_{W^{1,2}}^{2}\right)\right), \\
t \in[0, T] .
\end{array}
$$

Step 2. From the analysis in Step $1,\|u(t)\|_{L^{2}}$ is bounded in $[0, T]$. Furthermore, by using Lemma 9 , for any $p<2 n /(n-2)$ and small enough constant $\tau>0$, there is a constant $c$ such that $\|\nabla w(t)\|_{L^{p}} \leq c$ for all $t \in[\tau, T]$ since $w(x, t) \in$ $C^{2,1}(\Omega ;(\tau, T]) \cap C\left((\tau, T] ; C^{3}(\Omega)\right)$. Applying the operator $\nabla$ on both sides of the second equation of (1),

$$
\frac{\partial \nabla w}{\partial t}=\nabla(\Delta w)-\nabla w+\nabla u
$$

Taking the inner product of (42) with $-\nabla(\Delta w)$, for any $\varepsilon_{2}>0$,

$$
\begin{aligned}
& \frac{1}{2} \frac{d\|\Delta w\|_{L^{2}}^{2}}{d t} \\
& \quad+\int_{\Omega}|\nabla(\Delta w)|^{2} d x+\int_{\Omega}|\Delta w|^{2} d x=-\int_{\Omega} \nabla u \cdot \nabla(\Delta w) d x \\
& \quad \leq \frac{\varepsilon_{2}^{2}}{2} \int_{\Omega}|\nabla(\Delta w)|^{2} d x+\frac{1}{2 \varepsilon_{2}^{2}} \int_{\Omega}|\nabla u|^{2} d x .
\end{aligned}
$$

From (32) and (43), there exist $\varepsilon=1 / 4, \varepsilon_{2}=\sqrt{2}$, and a constant $c_{\varepsilon, \varepsilon_{2}}$ depending only on $a, b,|\Omega|$ such that

$$
\frac{1}{2}\left(\frac{d\|u(t)\|_{L^{2}}^{2}}{d t}+\frac{d\|\Delta w\|_{L^{2}}^{2}}{d t}\right)+\frac{1}{8}\|\nabla u\|_{L^{2}}^{2}+\frac{7}{8}\|\Delta w\|_{L^{2}}^{2} \leq c_{\varepsilon, \varepsilon_{2}} .
$$

By Poincaré inequality and Gronwall's lemma, then

$$
\begin{array}{r}
\|u\|_{L^{2}}^{2}+\|\Delta w\|_{L^{2}}^{2} \leq c\left(2 c_{\varepsilon, \varepsilon_{2}}+e^{(-1 / 8) t}\left(\left\|u_{\tau}\right\|_{L^{2}}^{2}+\left\|\Delta w_{\tau}\right\|_{L^{2}}^{2}\right)\right), \\
t \in(\tau, T]
\end{array}
$$

Taking the inner product of the first equation of (1) with $-\Delta u$ in $L^{2}(\Omega)$, then

$$
\begin{aligned}
& \frac{1}{2} \frac{d\|\nabla u\|_{L^{2}}^{2}}{d t} \\
& \quad+\int_{\Omega}|\Delta u|^{2} d x=\int_{\Omega} \nabla \cdot\left(u^{\alpha} \nabla g(w)\right) \Delta u d x \\
& \quad+\int_{\Omega} f^{\prime}(u)|\nabla u|^{2} d x \\
& \leq \frac{\varepsilon_{3}^{2}}{2} \int_{\Omega}|\Delta u|^{2} d x+\frac{1}{2 \varepsilon_{3}^{2}} \int_{\Omega}\left|\nabla \cdot\left(u^{\alpha} \nabla g(w)\right)\right|^{2} d x \\
& \quad+a \int_{\Omega}|\nabla u|^{2} d x .
\end{aligned}
$$

Since $g(s)$ is a real smooth function and $\left|g^{\prime}(s)\right|<\lambda$ for all $s \in$ $[0,+\infty)$, then there is a constant $M>0$, such that $\left|g^{\prime \prime}(s)\right| \leq$ $M$ and

$$
\begin{aligned}
& \left|\nabla \cdot\left((u+1)^{\alpha} \nabla g(w)\right)\right|^{2} \\
& =\left.\left|\alpha g^{\prime}(w)(u+1)^{\alpha-1} \nabla u \cdot \nabla w+g^{\prime \prime}(w)(u+1)^{\alpha}\right| \nabla w\right|^{2} \\
& \quad+\left.g^{\prime}(w)(u+1)^{\alpha} \Delta w\right|^{2} \\
& \quad \leq c\left[|\nabla u \cdot \nabla w|^{2}+(u+1)^{2 \alpha}|\Delta w|^{2}+(u+1)^{2 \alpha}|\nabla w|^{4}\right] .
\end{aligned}
$$

By Hölder's inequality,

$$
\int_{\Omega}|\nabla u \cdot \nabla w|^{2} d x \leq\|\nabla u\|_{L^{2 p}}^{2}\|\nabla w\|_{L^{2 q}}^{2},
$$

where $1 / p+1 / q=1$. There is $p \in(n / 2, n /(n-2))$ for $n=2,3$ such that $2 q<2 n /(n-2), 2 p(n-2)<2 n$. By using Lemma 9 , there is a constant $c$ such that $\|\nabla w\|_{L^{4}} \leq c$. Then from Lemmas 3 and 7 and the Poincaré inequality, for any $\varepsilon_{0}>0$, there exists a constant $c_{\varepsilon_{0}}$ such that

$$
\begin{aligned}
& \|\nabla u\|_{L^{2 p}}^{2} \\
& \quad \leq\|\nabla u\|_{W^{1,2}}^{2 a}\|\nabla u\|_{L^{2}}^{2(1-a)} \leq c\|u\|_{H^{2}}^{2 a}\|u\|_{H^{1}}^{2(1-a)} \\
& \quad \leq c\|u\|_{H^{2}}^{2 a}\left(\|u\|_{H^{2}}^{1 / 2}\|u\|_{L^{2}}^{1 / 2}\right)^{2(1-a)} \\
& \quad \leq \varepsilon_{0}\|u\|_{H^{2}}^{2}+c_{\varepsilon_{0}}\|u\|_{L^{2}}^{2},
\end{aligned}
$$

where $a=n / 2-n / 2 p<1$

Using the same method as the above analysis, for any $p_{1}$ satisfying $p_{1}>n / 2,2 p_{1} \alpha \leq 2$, and $p_{1}^{\prime}<n /(n-2)$, there exist 
$a=n / 2-n / 2 p_{1} \in(0,1)$ and arbitrary constant $\delta>0$ such that

$$
\begin{gathered}
\int_{\Omega}(u+1)^{2 \alpha}|\Delta w|^{2} d x \leq\|u+1\|_{L^{2 p_{1} \alpha}}^{2 \alpha}\|\Delta w\|_{L^{2 p_{1}^{\prime}}}^{2}, \\
\|\Delta w\|_{L^{2 p_{1}^{\prime}}}^{2} \leq\|\Delta w\|_{H^{1}}^{2 a}\|\Delta w\|_{L^{2}}^{2(1-a)} \leq \delta\|\nabla \Delta w\|_{L^{2}}^{2}+c_{\delta}\|w\|_{H^{2}}^{2} .
\end{gathered}
$$

Equation (45) implies that $\|\Delta w\|_{L^{2}}$ and $\|u\|_{L^{2}}$ are bounded, and then there exists a constant $c$ such that $\|u+1\|_{L^{2 p \alpha}}^{2 \alpha} \leq$ $\|u+1\|_{L^{2}}^{2 \alpha} \leq c$ and $\int_{\Omega}(u+1)^{2 \alpha}|\Delta w|^{2} d x \leq c \delta\|\nabla \Delta w\|_{L^{2}}^{2}+$ $c c_{\delta}\|w\|_{H^{2}}^{2}$.

By Lemma 9 and $\alpha<1 / n$, for any $p_{2}$ satisfying $p_{2}>n /(4-$ $n), 2 p_{2} \alpha \leq 2$, and $4 p_{2}^{\prime}<2 n /(n-2)$, there exists a constant $c$ such that

$$
\begin{aligned}
\int_{\Omega}(u+1)^{2 \alpha}|\nabla w|^{4} d x & \leq\|u+1\|_{L^{2 p_{2} \alpha}}^{2 \alpha}\|\nabla w\|_{L^{4 p_{2}^{\prime}}}^{4} \\
& \leq\|u+1\|_{L^{2}}^{2}\|\nabla w\|_{L^{4 p_{2}^{\prime}}}^{4} \leq c
\end{aligned}
$$

where $1 / p_{1}+1 / p_{1}^{\prime}=1$

From the above analysis, if $\varepsilon_{2}=1, \varepsilon_{3}=1$ in (43), (46) and $\varepsilon_{0}, \delta$ are small enough, then there exists positive constant $v>0$ such that

$$
\begin{aligned}
& \frac{1}{2} \frac{d\left(\|\Delta w\|_{L^{2}}^{2}+\|\nabla u\|_{L^{2}}^{2}\right)}{d t} \\
& \quad+\int_{\Omega}|\nabla(\Delta w)|^{2} d x \\
& \quad+v \int_{\Omega}\left(|\Delta w|^{2}+|\nabla u|^{2}\right) d x \leq c\left(\|u\|_{L^{2}},\|w\|_{H^{1}}\right) .
\end{aligned}
$$

By Gronwall's lemma,

$$
\begin{array}{r}
\|w\|_{H^{2}}+\|u\|_{H^{1}} \leq c\left[c\left(\varepsilon, \varepsilon_{1}\right)+e^{-v t}\left(\left\|w_{\tau}\right\|_{H^{2}}+\left\|u_{\tau}\right\|_{H^{1}}\right)\right] \\
t \in(\tau, T] .
\end{array}
$$

Equations (45), (53), and the choice of $T$ (in Theorem 10) depending on $\left\|u_{0}\right\|_{L^{2}(\Omega)}+\left\|w_{0}\right\|_{W^{1,2}(\Omega)}$ imply that $(u(x, T), w(x, T)) \in S$. It is clear by a standard argument that the solution $(u, w)$ to (1) can be extended up to some $T_{\max } \leq \infty$. With the same method as in the proof of Lemma 12 , for any finite $T_{\max }$,

$$
\begin{gathered}
\left\|u\left(T_{\max }\right)\right\|_{L^{2}}^{2} \\
+\left\|w\left(T_{\max }\right)\right\|_{W^{1,2}}^{2} \leq c\left(1+e^{-v T_{\max }}\left(\left\|u_{0}\right\|_{L^{2}}^{2}+\left\|w_{0}\right\|_{W^{1,2}}^{2}\right)\right) \\
\left\|u\left(T_{\max }\right)\right\|_{H^{1}}+\left\|w\left(T_{\max }\right)\right\|_{H^{2}} \leq C
\end{gathered}
$$

which implies that $T_{\max }=+\infty$. The global existence of the solution to (1) is obtained as the following theorem.
Theorem 13. Suppose that nonnegative functions $u_{0} \in L^{2}(\Omega)$, $w_{0} \in W^{1,2}(\Omega)$, and $\alpha<1 / n$, and then there is a unique nonnegative global solution $(u, w)$ to $(1)$ satisfying

$$
\begin{gathered}
u \in C\left([0, \infty) ; L^{2}(\Omega)\right) \bigcap C^{1}\left((0, \infty) ; L^{2}(\Omega)\right) \\
\bigcap C\left((0, \infty) ; H^{1}(\Omega)\right), \\
w \in C\left([0, \infty) ; W^{1,2}(\Omega)\right) \bigcap C^{1}\left((0, \infty) ; W^{1,2}(\Omega)\right) \\
\bigcap C\left((0, \infty) ; H^{2}(\Omega)\right), \\
\|u(t)\|_{L^{2}}^{2}+\|w(t)\|_{W^{1,2}}^{2} \leq c\left(1+e^{-v t}\left(\left\|u_{0}\right\|_{L^{2}}^{2}+\left\|w_{0}\right\|_{W^{1,2}}^{2}\right)\right), \\
\|u(t)\|_{H^{1}}+\|w(t)\|_{H^{2}} \leq C, \quad t>T_{1}+1,
\end{gathered}
$$

where $C$ depends only on $\Omega, u_{T_{1}}, w_{T_{1}}$, and $T_{1}=$ $(1 / v) \ln \left(\left\|u_{0}\right\|_{L^{2}}^{2}+\left\|w_{0}\right\|_{W^{1,2}}^{2}\right)$.

Remark 14. From the estimates in Lemma 12, there exists fixed constant $M>0$ and $T_{1}=(1 / v) \ln \left(\left\|u_{0}\right\|_{L^{2}}^{2}+\left\|w_{0}\right\|_{W^{1,2}}^{2}\right)$ such that

$$
\|u(t)\|_{L^{2}}+\|w(t)\|_{W^{1,2}} \leq M, \quad t>T_{1} .
$$

Denote the set $B=\left\{(u, w) \in L^{2}(\Omega) \times H^{1}(\Omega):\|u, w\|_{L^{2} \times H^{1}} \leq\right.$ $M ; u \geq 0, w \geq 0\}$, where $M$ is the constant in (56). The results of Theorem 13 imply that the existence of a dynamical system $\{S(t)\}_{t \geq 0}$ which maps $V=\left\{(u, w):(u, w) \in L^{2} \times\right.$ $\left.H^{1} ; u \geq 0, w \geq 0\right\}$ into itself and satisfying $(u(t), w(t))=$ $S(t)\left(u_{0}, w_{0}\right)$. Since $B$ is bounded, by Lemma 12 , there exists $T(B)$ depending only on $B$ and $|\Omega|$ such that

$$
S(t) B \subset B, \quad t \geq T(B)
$$

which implies that $B$ is a bounded absorbing set of the semi$\operatorname{group}\{S(t)\}_{t \geq 0}$.

Next, by the Sobolev embedding theorem, the asymptotical compactness of the semigroup $\{S(t)\}_{t \geq 0}$ is shown, and then the existence of a global attractor to system (1) is given.

Theorem 15. Assume that $\alpha<1 / n$. Then the problem (1) has a global attractor which is a compact invariant set and attracts every bounded set in $V$.

Proof. If $\left(u_{n}, w_{n}\right)$ is bounded in $V$, assume that there exists $R$ such that $\left\|\left(u_{n}, w_{n}\right)\right\|_{V} \leq R$. Then by Lemma 12 , there is a constant $T_{1}(R)$ (depending on $R$ ) such that

$$
\left(u^{n}(t), w^{n}(t)\right)=S(t)\left(u_{n}, w_{n}\right) \subset B, \quad t \geq T_{1}(R),
$$

where $B$ is the absorbing set given in (57) and $n=1,2, \ldots$ For any sequence $t_{n}\left(t_{n} \rightarrow+\infty\right.$ as $\left.n \rightarrow \infty\right)$, there exists $N$ such that, for any $n>N(R), t_{n} \geq T_{1}(R)+1$,

$$
\begin{aligned}
& \left(u^{n}\left(t_{n}\right), w^{n}\left(t_{n}\right)\right) \\
& \quad=S\left(t_{n}-T_{1}(R)-1\right)\left(S\left(T_{1}(R)+1\right)\left(u_{n}, w_{n}\right)\right) .
\end{aligned}
$$


Since the two embedding $H^{1}(\Omega) \hookrightarrow L^{2}(\Omega), H^{2}(\Omega) \hookrightarrow$ $H^{1}(\Omega)$ are compact, from the estimates in Theorem 13, it is clear that $\left\{u^{n}\left(t_{n}\right)\right\}$ lies in a compact set in $L^{2}(\Omega)$ and $\left\{w^{n}\left(t_{n}\right)\right\}$ lies in a compact set in $H^{1}(\Omega)$. Hence, $\left\{\left.S\left(t_{n}\right)\left(u_{n}, w_{n}\right)\right|_{\Omega}\right\}$ is precompact in $L^{2}(\Omega) \times H^{1}(\Omega)$, which implies that $\{S(t)\}_{t \geq 0}$ is asymptotically compact. In Remark 14, we have obtained the bounded absorbing set of $\{S(t)\}_{t \geq 0}$. Then by Proposition 1, we obtain the existence of the global attractor to (1).

\section{Acknowledgments}

This paper is supported by the National Natural Science Foundation of China (no. 11272277), Program for New Century Excellent Talents in University (NCET-10-0238), the Key Project of Chinese Ministry of Education (no. 211105) and Innovation Scientists and Technicians Troop Construction Projects of Henan Province (134100510013), Innovative Research Team in University of Henan Province (13IRTSTHN019), Foundation of Henan Educational Committee (no. 13A110737), and Hall of Henan Province Science and Technology Project (no. 12A110020).

\section{References}

[1] D. Horstmann and M. Winkler, "Boundedness vs. blow-up in a chemotaxis system," Journal of Differential Equations, vol. 215, no. 1, pp. 52-107, 2005.

[2] K. Osaki, T. Tsujikawa, A. Yagi, and M. Mimura, "Exponential attractor for a chemotaxis-growth system of equations," Nonlinear Analysis. Theory, Methods \& Applications, vol. 51, no. 1, pp. 119-144, 2002.

[3] X. Chen and W. Liu, "Global attractor for a density-dependent sensitivity chemotaxis model," Acta Mathematica Scientia B, vol. 32, no. 4, pp. 1365-1375, 2012.

[4] L. E. Payne and B. Straughan, "Decay for a Keller-Segel chemotaxis model," Studies in Applied Mathematics, vol. 123, no. 4, pp. 337-360, 2009.

[5] R. A. Quinlan and B. Straughan, "Decay bounds in a model for aggregation of microglia: application to Alzheimer's disease senile plaques," Proceedings of The Royal Society of London A, vol. 461, no. 2061, pp. 2887-2897, 2005.

[6] O. Ladyzhenskaya, Attractors for Semigroups and Evolution Equations, Cambridge University Press, Cambridge, UK, 1991.

[7] A. Pazy, Semigroups of Linear Operators and Applications to Partial Differential Equations, vol. 44 of Applied Mathematical Sciences, Springer, New York, NY, USA, 1983.

[8] J. K. Hale, Asymptotic Behavior of Dissipative Systems, vol. 25 of Mathematical Surveys and Monographs, American Mathematical Society, Providence, RI, USA, 1988.

[9] A. V. Babin and M. I. Vishik, "Attractors of partial differential evolution equations in an unbounded domain," Proceedings of the Royal Society of Edinburgh A, vol. 116, no. 3-4, pp. 221-243, 1990.

[10] A. Friedman, Partial Differential Equations, Holt Rinehart and Winston, New York, NY, USA, 1969.

[11] X. Mora, "Semilinear parabolic problems define semiflows on $C^{k}$ spaces," Transactions of the American Mathematical Society, vol. 278, no. 1, pp. 21-55, 1983. 


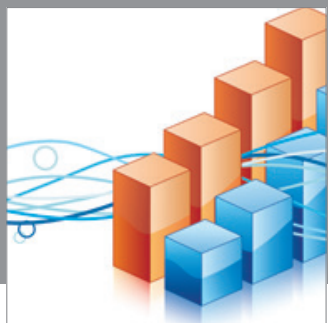

Advances in

Operations Research

mansans

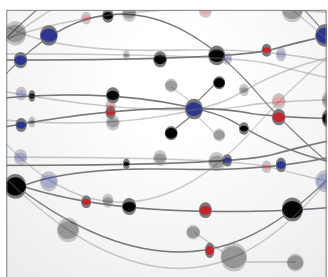

The Scientific World Journal
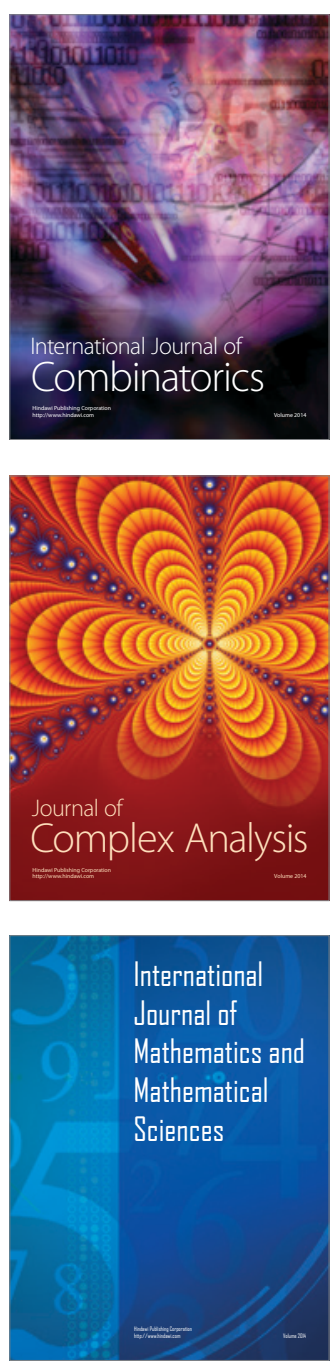
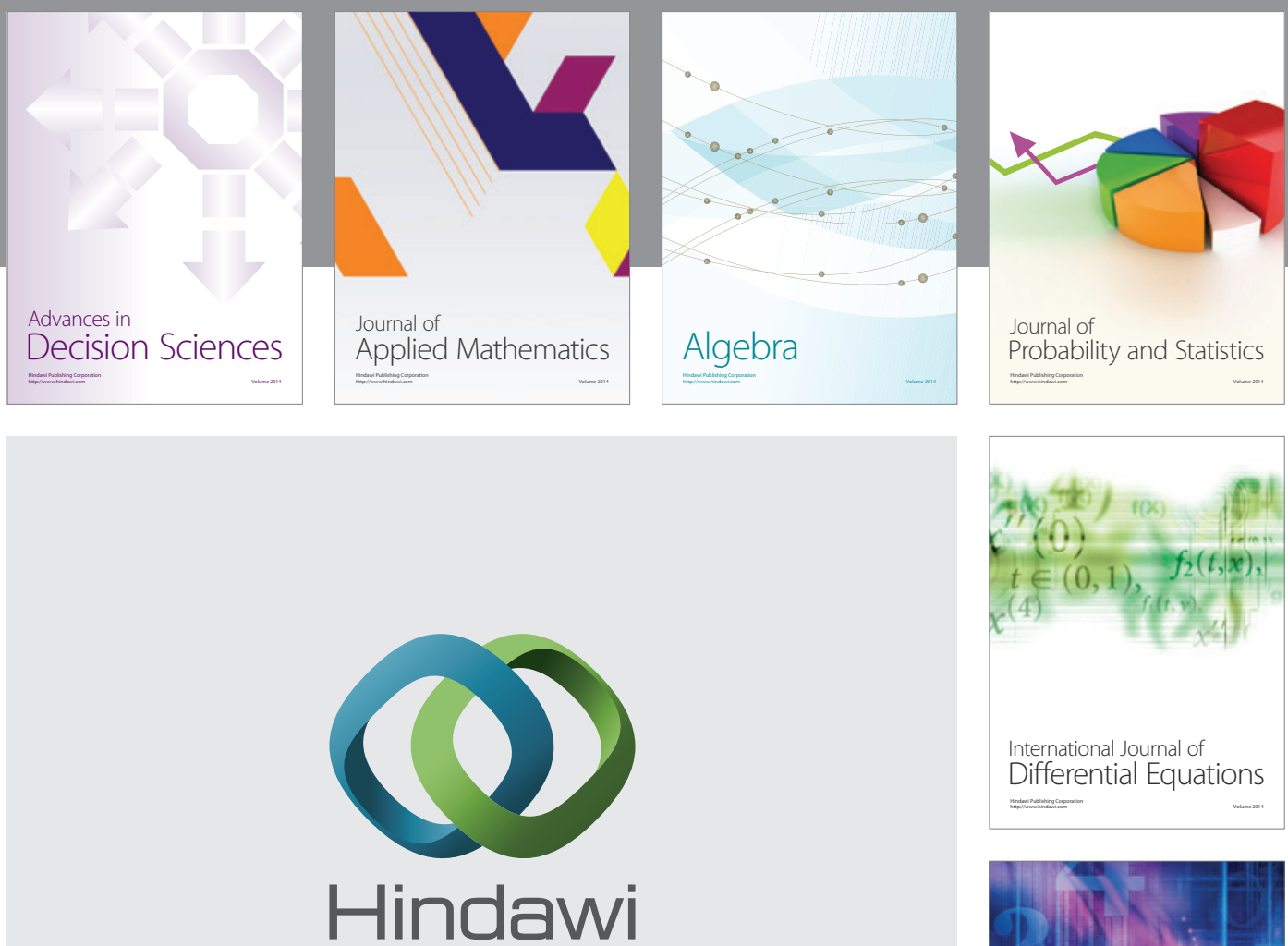

Submit your manuscripts at http://www.hindawi.com
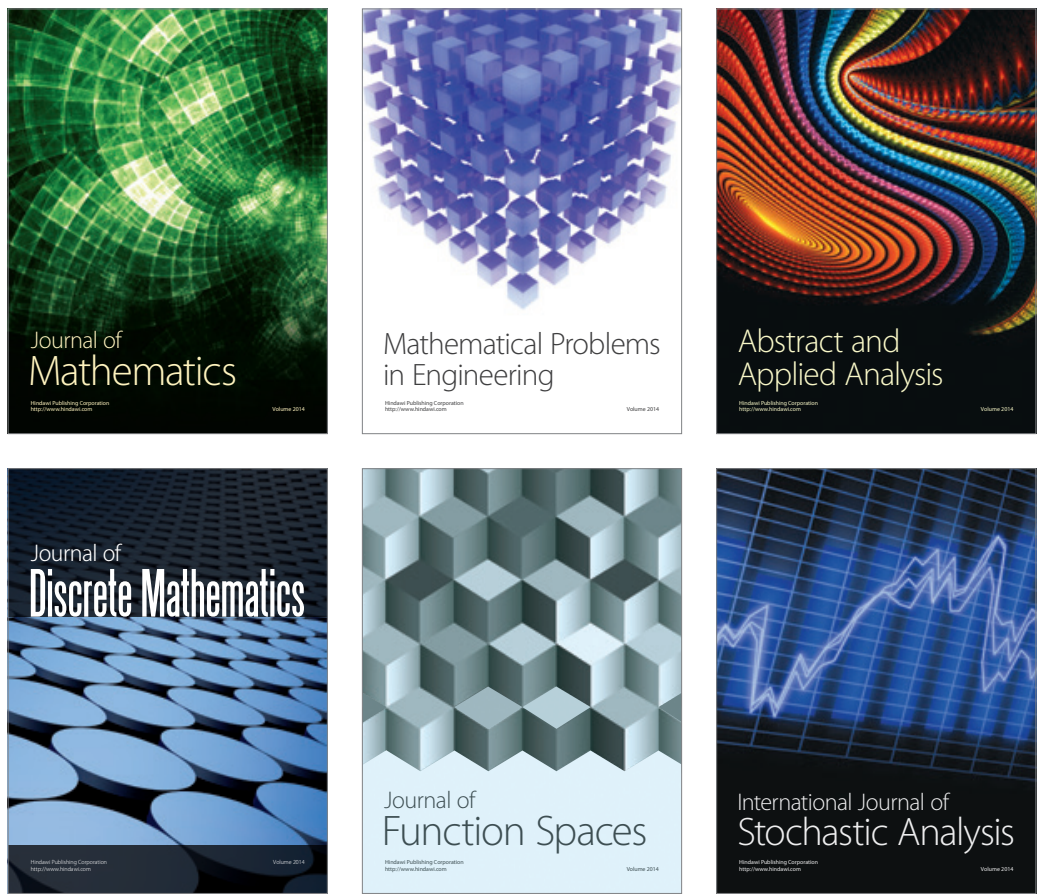

Journal of

Function Spaces

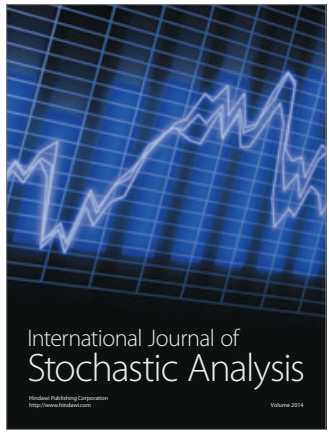

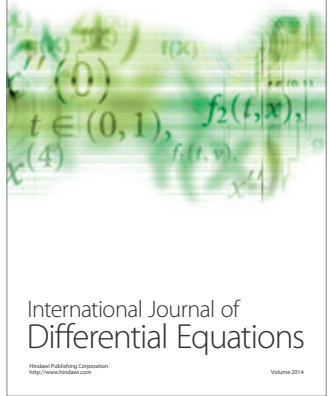
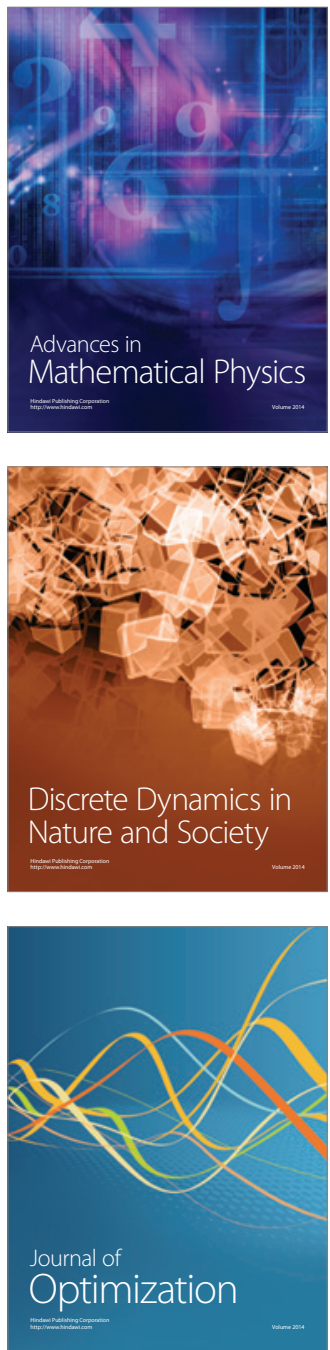